\title{
Effective area spectral efficiency for wireless communication networks with interference management
}

\author{
Aymen Omri ${ }^{1 *}$, Mazen O. Hasna ${ }^{1}$ and Mohammed Nafie 2,3
}

\begin{abstract}
In this paper, we introduce a new metric, namely, effective area spectral efficiency (EASE), to quantify the spectral efficiency as well as the spatial properties of point-to-point transmission systems and decode and forward (DF) relaying communications networks with interference management. For each transmission mode, we derive a closed-form expression for the maximum transmission range under Rayleigh fading environment. Based on the maximum transmission range, we define and derive the average affected area and the average ergodic capacity. We then introduce the EASE expression to quantify the spatial spectral utilization efficiency. For DF relaying, the EASE metric is based on a newly introduced index, namely, source relay communication index (SRCndx). SRCndx is used to validate the communication possibility between a source and a relay for given transmission parameters in a given environment and provides information about the necessity of using relaying communications. Through mathematical analysis and numerical examples, we show that the EASE metric provides a new perspective on the design of wireless transmissions, especially the transmission power selection process.
\end{abstract}

Keywords: Affected area; Link reliability; Spectral efficiency; Wireless communication networks

\section{Introduction}

The primary goal of new wireless communication systems is to support high-data-rate transmissions over limited radio spectrum with minimum amount of power consumption [1,2]. For this reason, development of new transmission schemes that can be operated in a more energy-efficient fashion has drawn much research attention [3-6].

To evaluate and compare the efficiency of the proposed communications schemes, effective performance metrics that can characterize the efficiency of radio spectrum and energy resources are needed $[3,4]$.

\section{Related work}

In wireless communication systems, the most conventional performance metrics focus on the quantification of either link reliability or spectral efficiency [5-8]. The link reliability is usually quantified in terms of outage

\footnotetext{
*Correspondence: omriaymen@qu.edu.qa

1 Electrical Engineering Department, Qatar University, Doha, Qatar

Full list of author information is available at the end of the article
}

probability or average error rate $[5,6]$, while the ergodic capacity addresses the spectral efficiency of wireless links, defined as the maximum achievable average spectrum efficiency [7, 8]. In the system/network level, european telecommunications standards institute (ETSI) defines the energy efficiency metric as the ratio of coverage area over power consumption at the base station [9]. Recently, in [10], the metric area power consumption $\left(\mathrm{W} / \mathrm{km}^{2}\right)$ for cellular networks is introduced to optimize base station deployment strategies. Bit per Joule (bit/J) is another metric, defined as the ratio of achievable rate over power consumption, and is used to quantify the energy utilization efficiency of emerging wireless systems [11]. Recently, this performance metric was applied to the analysis of coordinated multipoint (CoMP) cellular systems as well as heterogeneous networks [12, 13]. However, this metric is still short of taking into account the spatial effect of wireless transmissions. Area spectral efficiency (ASE) performance metric was presented in [14] to quantify the spectrum utilization efficiency of cellular systems. ASE is defined as the maximum data rate per unit bandwidth for

\section{Springer}

(C) 2015 Omri et al. Open Access This article is distributed under the terms of the Creative Commons Attribution 4.0 International License (http://creativecommons.org/licenses/by/4.0/), which permits unrestricted use, distribution, and reproduction in any medium, provided you give appropriate credit to the original author(s) and the source, provide a link to the Creative Commons license, and indicate if changes were made. 
a given user over cell coverage area where the user is randomly located, with a unit of $\left(\mathrm{bps} / \mathrm{Hz} / \mathrm{m}^{2}\right)$. As co-channel cells in cellular system are separated by a minimum reuse distance of $D$, the same spectrum will be used only once over an area of size $\frac{\pi D^{2}}{4}$. Note that the area used in ASE definition is based on the co-channel interference requirement of cellular system and is not related to the radio transmissions target. In [3, 15], and [16], a general area spectral efficiency (GASE) is defined as the average data rate per unit bandwidth and unit area supported by a base station. In [3], the authors have introduced the concept of affected area, based on which they evaluated the spectral and power efficiency of three different transmission scenarios, namely, single user point-to-point transmissions, two-user $\mathrm{X}$ channel transmissions, and two-hop relay transmissions. In [15] and [16], the GASE is used to evaluate the performance of cooperative networks; however, since there is no such central base station (BS), the authors have redefined GASE to be the average data rate per unit bandwidth within a particular area, in which simultaneous transmissions are not allowed. The disadvantage of the introduced GASE metric in $[3,15]$, and [16] is that it was calculated by using either the cell area covered by the base station (BS) or the relay station, then averaging the GASE values of the two areas. In addition, for direct link (DL) transmission, the GASE was calculated for a given distance between the BS and the user. For cooperative transmission, on the other hand, the GASE was calculated for a given distance between the relay and the user. This makes the GASE a metric for a given user position and not for all the area covered by the communication systems. In addition, the optimization of the GASE with respect to the transmission power was presented without power constraints. As we will show later in the simulation results, without transmission power constraints, there are some power values for a given distance between a source and a destination where the communication cannot be completed with respect to a minimum threshold received power. Hence, those values should not be used in the maximization of GASE, and this is not the case in $[3,15]$, and [16].

To prevent such kind of problems, we present in this paper a new robust and effective metric of area spectral efficiency for DL and DF communication systems.

\section{Contributions}

In this paper, we present and derive the maximum transmission range for arbitrary wireless communication systems based on given transmission parameters and through Rayleigh fading channels. Based on which, we compute the average affected area, and we present the new performance metric "EASE", which can be used to evaluate the spectrum utilization and the power utilization efficiencies of arbitrary wireless transmissions. We study and derive the EASE performance expression for DL transmissions and DF relaying mode with three different interference management schemes. The interference problem presented within this paper results when a user is receiving an undesirable signal from a relay selected to cooperate with other destinations. The first considered interference management scheme is based on the well known halfduplex relaying (HDR), when simultaneous transmissions from the source and the relay are not allowed. The second scheme, which is called interference constraint-based relay selection (ICRS), was introduced in [17]. The main idea in this scheme is that simultaneous transmissions from the source and the relay are allowed only if the user which is receiving a data from the source is far enough from the relay. The third scheme, best relay and user selection (BRUS) technique, was introduced in [5] to minimize the interference problems by using an optimal resources allocation between users. Here, the idea is to select the user which is far enough from the relay and allocate this user to the same time slot used by the relay to prevent the interference at this user.

For DF relaying transmissions, the EASE metric is based on a new introduced index, namely, SRCndx. SRCndx is used to validate the communication possibility between a source and a relay for given transmission parameters in a given environment and then provides information about the necessity of using relaying communications. Based on the performance comparison with GASE presented in $[3,15]$, and [16], and through the analytical results and numerical examples, we show that the EASE metric provides a new perspective on the design of wireless transmissions, especially on the transmission power selection.

\section{Organization of the paper}

The remainder of this paper is organized as follows: the "Effective area spectral efficiency for DL communications" section describes and derives the expression of the new metric EASE for DL transmissions. The expressions of EASE metric and SRCndx index for DF relaying communications with interference management schemes are derived in the "Effective area spectral efficiency for DF communications" section. The "Numerical results and interpretations" section presents the numerical results and interpretations. Finally, conclusions are drawn in the "Conclusions" section.

\section{Effective area spectral efficiency for DL communications}

In this section, we formally introduce the definition of EASE metric for DL communications. First, we present and derive the expression of maximum transmission range, based on which we derive the affected area and the average ergodic capacity. Then, we introduce the EASE metric expression. 


\section{Maximum transmission range}

For DL, the probability that a position with distance $r_{i}$ from the transmitter is "affected" is equal to the probability that a minimum received signal power $\left(P_{\text {min }}\right)$ is observed in this position. Mathematically, this probability can be expressed as follows:

$$
P\left(P_{r} \geq P_{\text {min }}\right)=P\left(\frac{P_{t} g}{r_{i}^{\alpha}} \geq P_{\text {min }}\right),
$$

where, $P_{t}$ is the transmission power, and $\alpha$ is the path loss exponent. Under Rayleigh fading environment, $g$ is an exponential random variable with unit mean. Based on (1), the instantaneous maximum transmission range is given by the following:

$$
r_{i}(g)=\left(\frac{P_{t} g}{P_{\min }}\right)^{\frac{1}{\alpha}} .
$$

We define the average maximum transmission range as the expectation $E\left\{r_{i}(g)\right\}$. By averaging $r_{i}(g)$ over the distribution of $g,(f(g))$, the average maximum transmission range can be calculated as follows:

$$
\begin{aligned}
r & =\int_{0}^{+\infty} r_{i}(g) f(g) d g \\
& =\left(\frac{P_{t}}{P_{\text {min }}}\right)^{\frac{1}{\alpha}} \int_{0}^{+\infty} g^{\frac{1}{\alpha}} \exp (-g) d g \\
& =\left(\frac{P_{t}}{P_{\text {min }}}\right)^{\frac{1}{\alpha}} \Gamma s\left(\frac{1}{\alpha}+1\right),
\end{aligned}
$$

where, $\Gamma(x)=\int_{0}^{+\infty} t^{x-1} \exp (-t) d t$ is the Gamma function [18].

\section{Affected area}

The affected area is defined as the area where a minimum received signal power $\left(P_{\min }\right)$ is observed. By using an omnidirectional transmitting antenna, the affected area is given by

$$
\begin{aligned}
A & =\pi r^{2} \\
& =\pi\left(\frac{P_{t}}{P_{\min }}\right)^{\frac{2}{\alpha}}\left(\Gamma\left(\frac{1}{\alpha}+1\right)\right)^{2} .
\end{aligned}
$$

\section{Average ergodic capacity}

For a given distance $r_{i}$ from the source, the ergodic capacity can be calculated as follows:

$$
\begin{aligned}
C_{D L} & =\int_{0}^{+\infty} \log _{2}\left(1+\frac{P_{t} g}{N r_{i}^{\alpha}}\right) f(g) d g \\
& =\frac{1}{\ln 2} E_{I}\left(\frac{N r_{i}^{\alpha}}{P_{t}}\right) \exp \left(\frac{N r_{i}^{\alpha}}{P_{t}}\right),
\end{aligned}
$$

where, $N$ is the additive Gaussian noise variance, and $E_{I}(x)=\int_{x}^{+\infty} t^{-1} \exp (-t) d t$ is the exponential integral [18]. As the mobile users are considered independent and uniformly distributed over the radius of the affected area, the average ergodic capacity expression can be written as

$$
\bar{C}_{D L}=\frac{1}{r \ln 2} \sum_{r_{i}=1}^{r} E_{I}\left(\frac{N r_{i}^{\alpha}}{P_{t}}\right) \exp \left(\frac{N r_{i}^{\alpha}}{P_{t}}\right) .
$$

Here, the summation is used rather than integration to make the expression more tractable. Finally, the EASE expression for DL transmissions is given by the following:

$$
E A S E=\frac{\sum_{r_{i}=1}^{r} E_{I}\left(\frac{N r_{i}^{\alpha}}{P_{t}}\right) \exp \left(\frac{N r_{i}^{\alpha}}{P_{t}}\right)}{\pi r \ln 2\left(\frac{P_{t}}{P_{\min }}\right)^{\frac{2}{\alpha}}\left(\Gamma\left(\frac{1}{\alpha}+1\right)\right)^{2}} .
$$

\section{Effective area spectral efficiency for DF relaying communications}

In this section, we derive the EASE expression for DF relaying communications. EASE is based on the total affected area by the source and the relay, the average ergodic capacity, and the SRCndx parameter that will be introduced later. In this work, we assume that the transmission power of the source, denoted by $P_{t}$, is higher than the transmission power of the relay which is denoted by $\mu P_{t}$, where $\mu$ is a predefined coefficient with a value less than one.

\section{Affected area}

Figure 1 shows the area covered by a source $S$ and a relay $R$. As shown in Fig. 1a, the total affected area, denoted by $\bar{A}$, is based on the distance $d_{S R}$, and the maximum transmission ranges of the source and the relay, denoted by $r_{S}$, and $r_{R}$, respectively.

$\bar{A}$ is calculated as follows:

$$
\bar{A}=A_{S}+A_{R}-\left(L_{S}+L_{R}\right),
$$

where, $A_{S}=\pi r_{S}^{2}$, and $A_{R}=\pi r_{R}^{2}$ are the areas covered by the source and the relay, respectively. $L_{S}$ is the lens area included in the covered area by the source. Based on Fig. $1 \mathrm{~d}, L_{S}$ is expressed as follows

$$
\begin{aligned}
L_{S} & =\int_{-\theta}^{\theta} \int_{0}^{r_{S}} r d r d \theta_{i}-z x \\
& =r_{S}^{2} \cos ^{-1}\left[\frac{r_{S}^{2}+d_{S R}^{2}-r_{R}^{2}}{2 r_{S} d_{S R}}\right]-z x .
\end{aligned}
$$

As shown in Fig. 1c, $L_{R}$ is a lens area that is included in the covered area by the relay. Similar to $L_{S}, L_{R}$ is calculated as follows:

$$
\begin{aligned}
L_{R} & =\int_{-\theta}^{\theta} \int_{0}^{r_{R}} r d r d \theta_{i}-z y \\
& =r_{R}^{2} \cos ^{-1}\left[\frac{r_{R}^{2}+d_{S R}^{2}-r_{S}^{2}}{2 r_{R} d_{S R}}\right]-z y .
\end{aligned}
$$



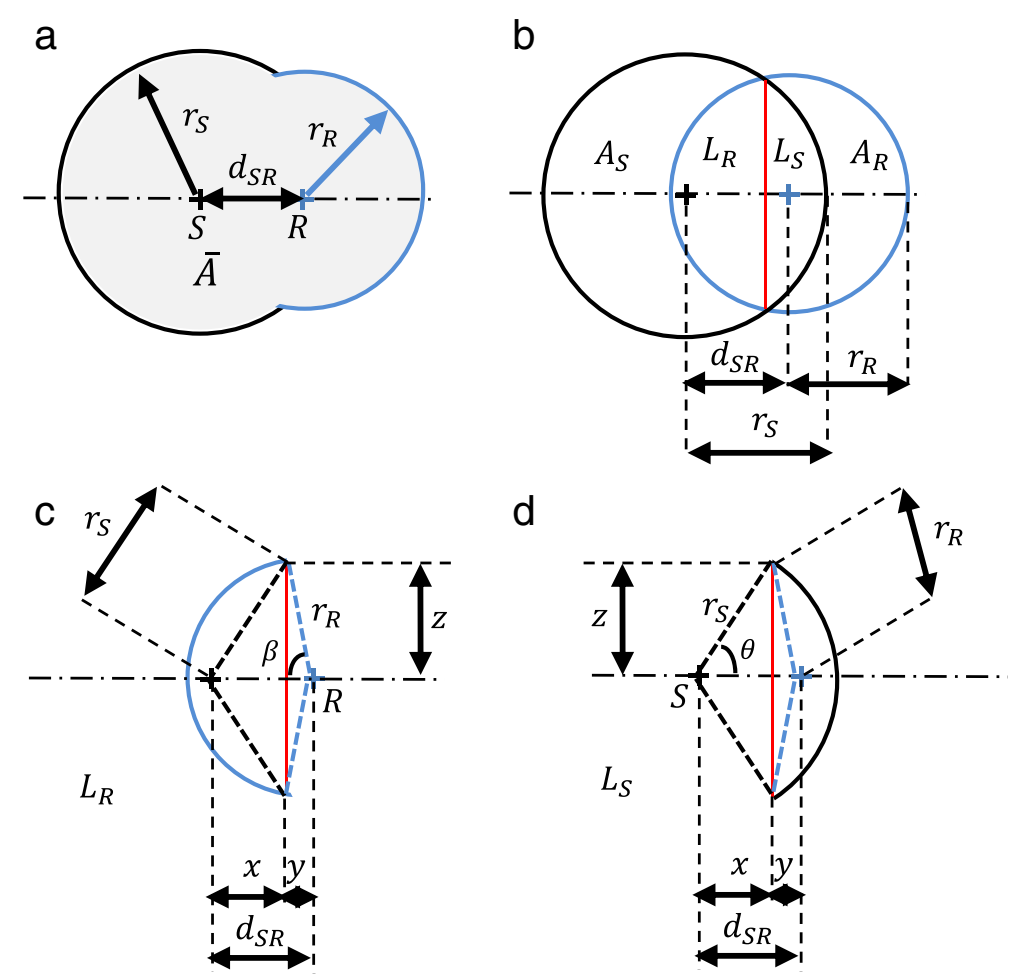

Fig. 1 The total affected area in DF relaying communications: a the total affected area, $\mathbf{b}$ the intersection of the covered area by the source and the covered area by the relay, $\mathbf{c}$ the lens area that is included in the covered area by the relay, $\mathbf{d}$ the lens area that is included in the covered area by the source

By using the following equality which is derived based on Fig. 1c

$$
\left[\frac{z}{r_{S}}\right]^{2}=\left[\frac{r_{S}^{2}+d_{S R}^{2}-r_{R}^{2}}{2 r_{S} d_{S R}}\right]^{2}
$$

the distance $z$ can be expressed as follows:

$$
z=\frac{1}{2 d_{S R}} \sqrt{r_{S}^{2}-\left[r_{S}^{2}+d_{S R}^{2}-r_{R}^{2}\right]^{2}}
$$

As $x+y=d_{S R}$, and after some simplifications, the total affected area is given by

$$
\begin{aligned}
\bar{A}= & \pi\left(r_{S}^{2}+r_{R}^{2}\right)-r_{S}^{2} \cos ^{-1}\left[\frac{r_{S}^{2}+d_{S R}^{2}-r_{R}^{2}}{2 r_{S} d_{S R}}\right] \\
& -r_{R}^{2} \cos ^{-1}\left[\frac{r_{R}^{2}+d_{S R}^{2}-r_{S}^{2}}{2 r_{R} d_{S R}}\right] \\
& +\frac{1}{2}\left[\left(r_{S}+r_{R}+d_{S R}\right)\left(r_{S}-r_{R}+d_{S R}\right)\left(r_{S}+r_{R}-d_{S R}\right)\right. \\
& \left.\times\left(-r_{S}+r_{R}+d_{S R}\right)\right]^{\frac{1}{2}} .
\end{aligned}
$$

\section{Source relay communication index (SRCndx)}

For some scenarios defined by given values of $d_{S R}, P_{t}, \alpha$, and $\mu$, there is no communication between the source and the relay, e.g., the communication scenarios presented in Figs. 2a and 3b. This is because the $P_{r}$ at the relay or the source is less than $P_{\min }$ due to the long distance between the two nodes. In other scenarios, when $d_{S R}+$ $r_{R}<r_{S}$, there is no need for the relay as its coverage is included in the source coverage e.g., the communication scenarios presented in Figs. 2c and 3c. To account for these scenarios, we present a new parameter, source relay communications index (SRCndx), which is given by the following:

$$
S R C n d x=\left\lceil\frac{1}{2}\left(\frac{r_{R}-d_{S R}}{\left|r_{R}-d_{S R}\right|}+\frac{r_{R}+d_{S R}-r_{S}}{\left|r_{R}+d_{S R}-r_{S}\right|}\right)\right\rceil,
$$

where, $\lceil$.$\rceil and |$.$| are the ceiling and the absolute$ value functions, respectively. As shown in (14), there are two main parts in the SRCndx parameter, the first part $\left(\frac{r_{R}-d_{S R}}{\left|r_{R}-d_{S R}\right|}\right)$ has a value of $(-1)$ if there is no communication between the source and the relay $\left(r_{R}<d_{S R}\right)$ and 1 if there is a possible communication between the source and the relay $\left(r_{R}>d_{S R}\right)$. The second part $\left(\frac{r_{R}+d_{S R}-r_{S}}{\left|r_{R}+d_{S R}-r_{S}\right|}\right)$ reduces to $(-1)$ if there is no need for relaying $\left(r_{R}+d_{S R}<r_{S}\right)$ and 1 if there is a need for relaying $\left(r_{R}+d_{S R}>r_{S}\right)$. Consequently, 

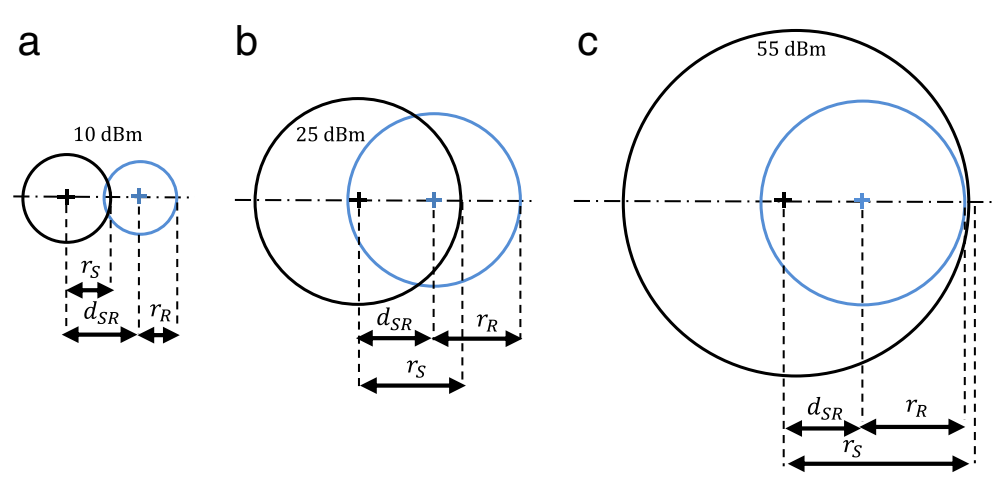

Fig. 2 Source relay communications scenarios for fixed $d_{S R}=500, P_{t}=10,25$, and $55 \mathrm{dBm}, \mu=0.5, \alpha=4$, and $P_{\min }=-90 \mathrm{dBm}$, a no communications scenario, $\mathbf{b}$ beneficial relaying scenario, $\mathbf{c}$ unuseful relaying scenario

by multiplying the sum of the two parts by $\frac{1}{2}$, we have the new parameter SRCndx which is 1 when there is communication between the source and the relay and there is a need for relaying, and 0 when there is no communication between the source and the relay or there is no need for relaying. This parameter will be used later on as part of the new metric EASE.

Figure 4 shows the variation of SRCndx versus $P_{t}$ for different radio propagation environments, with $d_{S R}=$ $500 \mathrm{~m}, \mu=0.5$, and $P_{\min }=-90 \mathrm{dBm}$. When there is no communication between the source and the relay, SRC$\mathrm{ndx}$ is equal to zero e.g., for $\alpha=4$, SRCndx $=0$ for $P_{t} \leq$ $20 \mathrm{dBm}$. It is equal to zero also when there is no need of relaying, e.g., for $\alpha=5$, SRCndx $=0$ for $P_{t} \geq 95 \mathrm{dBm}$. Where the relaying is beneficial for a specific range of $P_{t}$ and SRCndx is equal to one in that range. This range of power values gives the effective power domain of EASE metric.

Figure 5 shows the variation of SRCndx versus $d_{S R}$ for different radio propagation environments, with $P_{t}=$ $25 \mathrm{dBm}, \mu=0.5$, and $P_{\text {min }}=-90 \mathrm{dBm}$. Based on SRCndx, for $\alpha=4$, there is no communication between the source and the relay for $d_{S R} \geq 600 \mathrm{~m}$. Also there is no need of relaying for $\alpha=3$ when $d_{S R} \leq 1.25$ Km. Hence, for a given environment $\alpha, P_{t}, \mu$, and $P_{\text {min }}$,
SRCndx gives the range of $d_{S R}$ where relaying is beneficial. This range is the set of $d_{S R}$ values when SRCndx is equal to one.

Accordingly, for given transmission and environment parameters, and based on (14), SRCndx can be calculated and used to confirm if relaying is beneficial $(\mathrm{SRCndx}=1)$ or not $(\mathrm{SRCndx}=0)$. Based on that, and as we will show later, SRCdnx will be used in the EASE metric definition.

\section{Average ergodic capacity}

We present in this section the average ergodic capacity expressions for the different interference management schemes. Figure 6 shows the different transmissions scenarios for two users $U_{n}$ and $U_{n+1}$ that communicate in two successive time slots. Based on those scenarios, the average ergodic capacity of the two users for the different schemes are derived as follows.

\section{HDR scheme}

In this scheme, and to prevent interference, there are no simultaneous transmissions from the source and the relay. Relayed communications for each user will be used if the user is located in the area covered by the relay only. As the mobile users are considered mutually independent and uniformly distributed in the affected area, the average

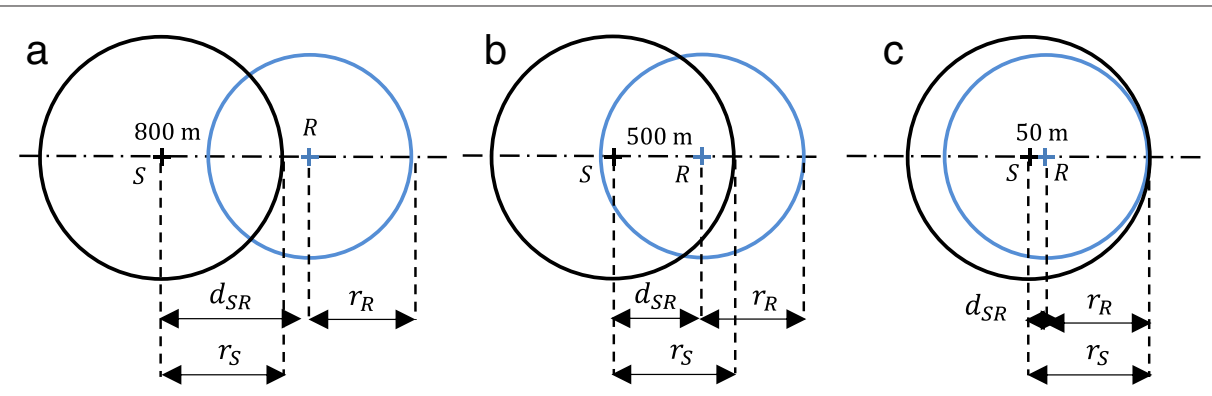

Fig. 3 Source relay communications scenarios for fixed $P_{t}=25 \mathrm{dBm}, d_{S R}=800,500$, and $50 \mathrm{~m}, \mu=0.5, \alpha=4, P_{\min }=-90 \mathrm{dBm}, r_{S}=287 \mathrm{~m}$, and $r_{R}=241 \mathrm{~m}$, a no communications scenario, b beneficial relaying scenario, c unuseful relaying scenario 

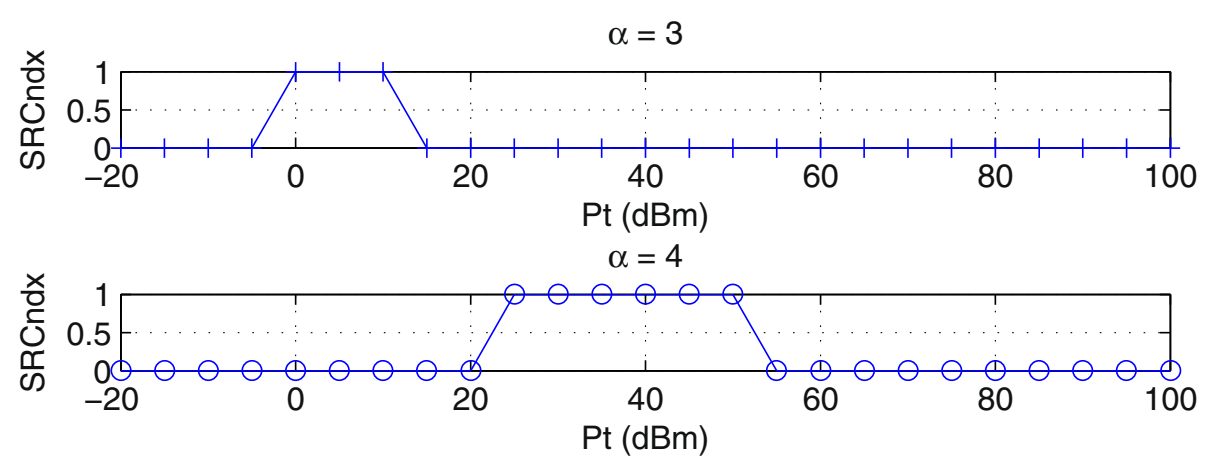

Pt $(\mathrm{dBm})$
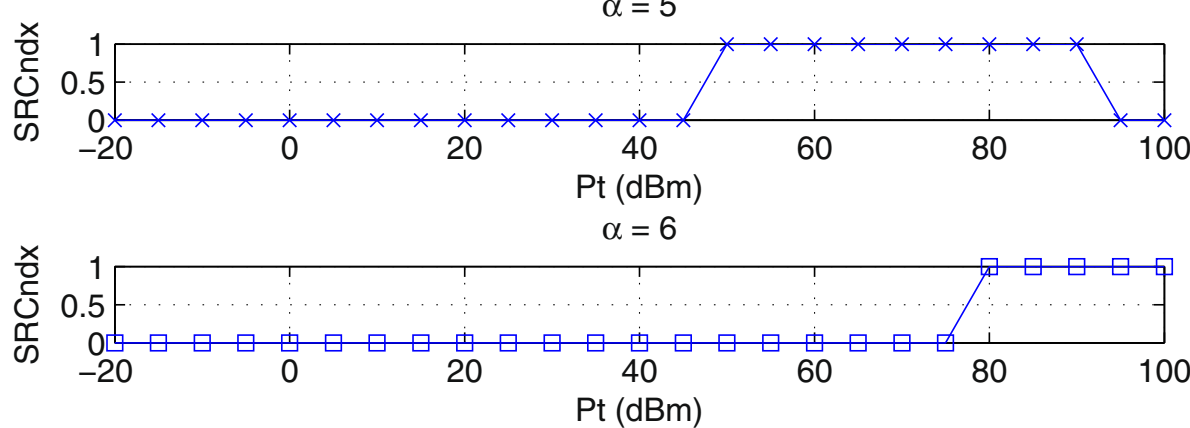

Fig. 4 Source relay communication index vs. $P_{t}$ for different radio propagation environments, with $d_{S R}=500 \mathrm{~m} \mu=0.5$, and $P_{\min }=-90 \mathrm{dBm}$
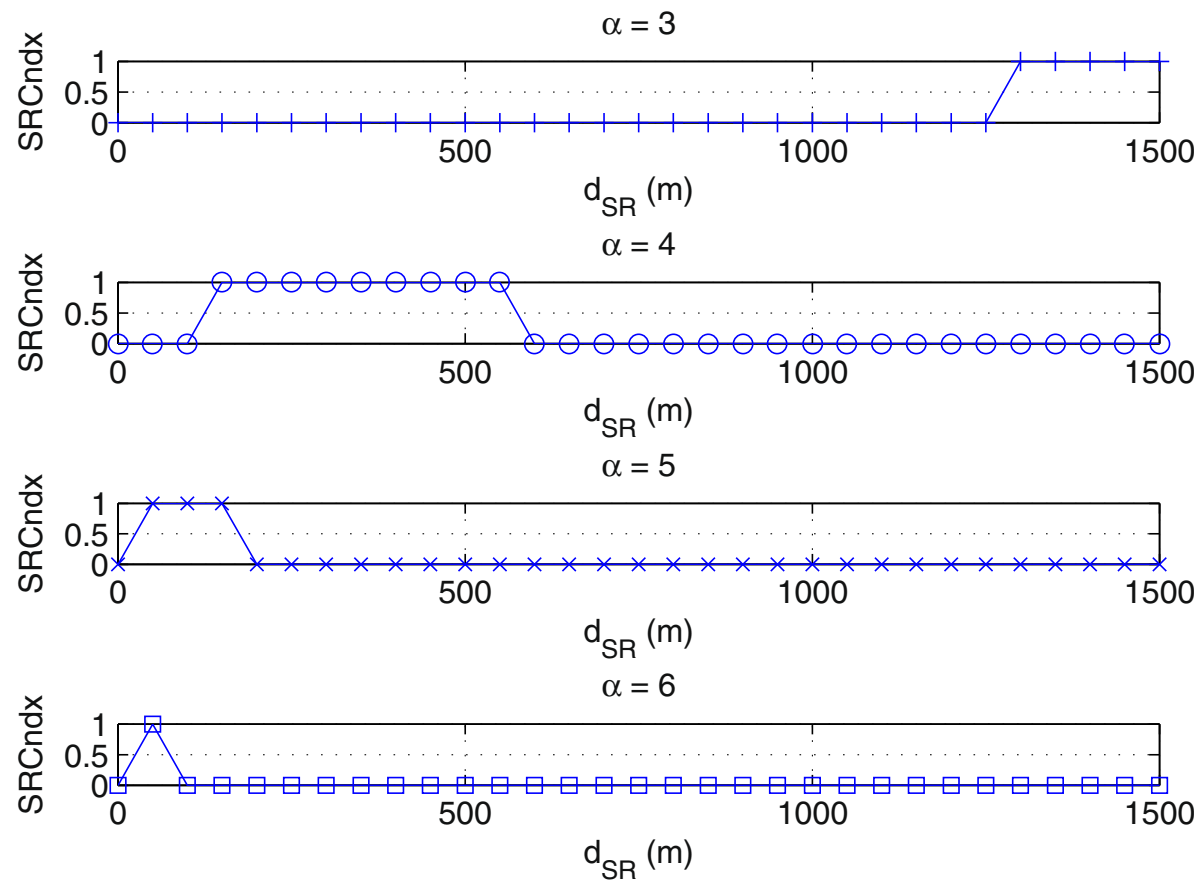

Fig. 5 Source relay communication index vs. $d_{S R}$ for different radio propagation environments, with $P_{t}=25 \mathrm{dBm}, \mu=0.5$, and $P_{\min }=-90 \mathrm{dBm}$ 


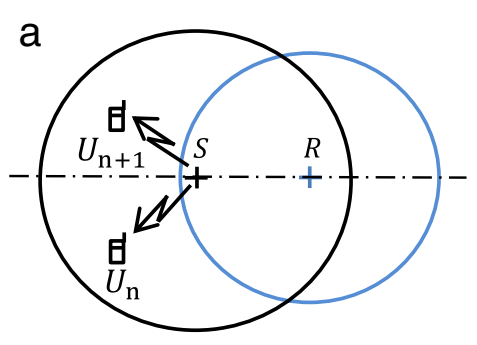

C

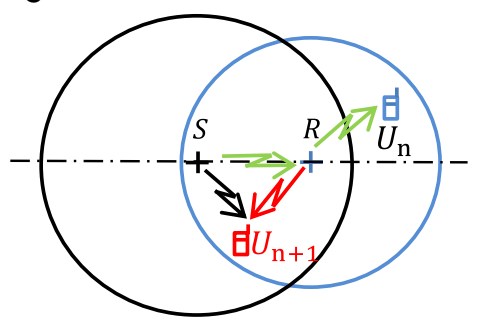

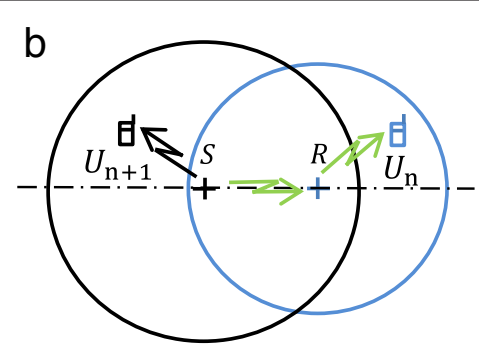

d

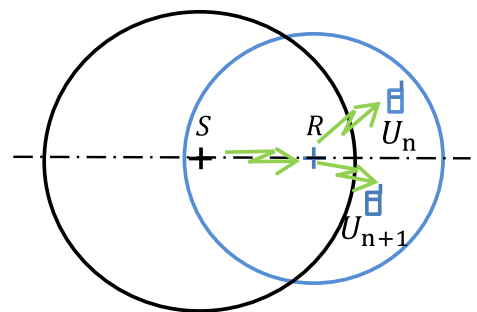

Fig. 6 The different transmissions scenarios based on users locations: $\mathbf{a}, \mathbf{b}$, and $\mathbf{d}$ no interference scenarios, $\mathbf{c}$ interference scenario

ergodic capacity for HDR scheme can be expressed as

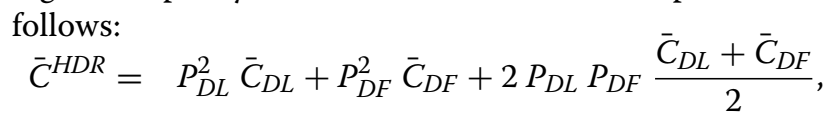

where, $P_{D L}=\frac{A_{S}}{\bar{A}}$ is the probability that a user is located in the source coverage area, $P_{D F}=\frac{A_{R}-\left(L_{S}-L_{R}\right)}{\bar{A}}$ is the probability that a user is located in the relay coverage area only, and $\bar{C}_{D F}$ is the average ergodic capacity for the DF relayed transmission. Based on Fig. 7, and the derivation of ergodic capacity for DF mode in [3], $\bar{C}_{D F}$ can be expressed as follows:

$$
\begin{aligned}
\bar{C}_{D F}= & \frac{1}{2 \ln 2 \sum_{\theta_{i}=0}^{\theta_{\max }} \sum_{r_{i}=r_{\min }}^{r_{R}} \sum_{\theta_{i}=0}^{\theta_{\max }} \sum_{r_{i}=r_{\min }}^{r_{R}} E_{I}\left(\frac{N d_{S R}^{\alpha}}{P_{t}}+\frac{N r_{i}^{\alpha}}{\mu P_{t}}\right)} \\
= & \frac{\exp \left(\frac{N d_{S R}^{\alpha}}{P_{t}}+\frac{N r_{i}^{\alpha}}{\mu P_{t}}\right)}{2 \ln 2\left(\theta_{\max }\left(r_{R}+1\right)-\sum_{\theta_{i}=0}^{\theta_{\max }} \sqrt{r_{S}^{2}+d_{S R}^{2}-2 r_{S} d_{S R} \cos \theta_{i}}\right)} \\
& \sum_{\theta_{i}=0}^{\theta_{\max }} \sum_{r_{i}=r_{\min }}^{r_{R}} E_{I}\left(\frac{N d_{S R}^{\alpha}}{P_{t}}+\frac{N r_{i}^{\alpha}}{\mu P_{t}}\right) \exp \left(\frac{N d_{S R}^{\alpha}}{P_{t}}+\frac{N r_{i}^{\alpha}}{\mu P_{t}}\right)
\end{aligned}
$$

where, $r_{\text {min }}=\sqrt{r_{S}^{2}+d_{S R}^{2}-2 r_{S} d_{S R} \cos \theta_{i}}$, and $\theta_{\max }=$ $\cos ^{-1}\left[\frac{r_{S}^{2}+d_{S R}^{2}-r_{R}^{2}}{2 r_{S} d_{S R}}\right]$.

\section{ICRS scheme}

The ICRS scheme was introduced in [17]. The transmission procedure within this scheme is based on the four scenarios that are presented in Fig. 6. For the scenarios presented in Fig. 6a, c, and d, ICRS uses the same transmission procedure as HD to prevent the interference caused by simultaneous transmissions at user $n$ or/and user $n+1$. In the case presented in Fig. $6 \mathrm{~b}$, the source and the relay can transmit simultaneously to users $n+1$ and $n$, respectively, without interfering with each other.

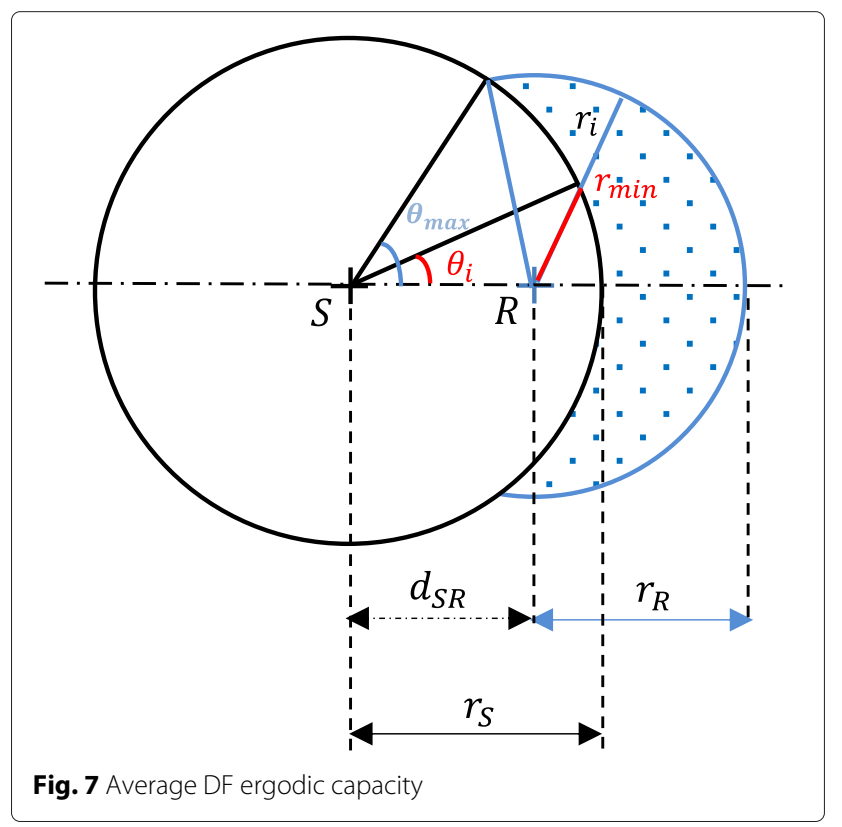


Hence, the average ergodic capacity for this scheme can be expressed as follows:

$$
\begin{aligned}
\hat{C}^{I C R S}= & P_{D L}^{2} \bar{C}_{D L}+P_{D F}^{2} \bar{C}_{D F}+2 P_{D L 1} P_{D F} \frac{\bar{C}_{D L 1}+2 \bar{C}_{D F}}{2} \\
& +2 P_{D L 2} P_{D F} \frac{\bar{C}_{D L 2}+\bar{C}_{D F}}{2}
\end{aligned}
$$

where, $P_{D L 1}=\frac{A_{S}-\left(L_{S}+L_{R}\right)}{\bar{A}}$ is the probability that a user is located in the area covered by the source only, and $P_{D L 2}=\frac{L_{S}+L_{R}}{\bar{A}}$ is the probability that a user is located in the area covered by the source and the relay. $\bar{C}_{D L 1}$ is the average ergodic capacity for the DL transmissions in the area covered by the source only, and $\bar{C}_{D L 2}$ is the average ergodic capacity for the DL transmissions in the area covered by the source and the relay. Based on Fig. 7 and (16), $\bar{C}_{D L 1}$ and $\bar{C}_{D L 2}$ can be expressed, respectively, as follows:

$$
\begin{aligned}
& \bar{C}_{D L 1}=\frac{1}{2 \ln 2 \sum_{\theta_{i}=0}^{\theta_{\max }^{\prime}} \sum_{r_{i}=r_{\text {min }}^{\prime}}^{r_{S}} 1} \sum_{\theta_{i}=0}^{\theta_{\max }^{\prime}} \sum_{r_{i}=r_{\text {min }}^{\prime}}^{r_{S}} E_{I}\left(\frac{N d_{S R}^{\alpha}}{P_{t}}+\frac{N r_{i}^{\alpha}}{\mu P_{t}}\right) \\
& \exp \left(\frac{N d_{S R}^{\alpha}}{P_{t}}+\frac{N r_{i}^{\alpha}}{\mu P_{t}}\right) \\
& =\frac{1}{2 \ln 2\left(\theta_{\max }^{\prime}\left(r_{S}+1\right)-\sum_{\theta_{i}=0}^{\theta_{\max }^{\prime}} \sqrt{r_{R}^{2}+d_{S R}^{2}-2 r_{R} d_{S R} \cos \theta_{i}}\right)} \\
& \sum_{\theta_{i}=0}^{\theta_{\max }^{\prime}} \sum_{r_{i}=r_{\text {min }}^{\prime}}^{r_{S}} E_{I}\left(\frac{N d_{S R}^{\alpha}}{P_{t}}+\frac{N r_{i}^{\alpha}}{\mu P_{t}}\right) \exp \left(\frac{N d_{S R}^{\alpha}}{P_{t}}+\frac{N r_{i}^{\alpha}}{\mu P_{t}}\right),
\end{aligned}
$$

and,

$$
\begin{aligned}
\bar{C}_{D L 2}= & \frac{1}{\ln 2\left(\sum_{\theta_{i}=0}^{2 \pi} \sum_{r_{i}=0}^{r_{S}} 1-\sum_{\theta_{i}=0}^{\theta_{\max }^{\prime}} \sum_{r_{i}=r_{\text {min }}^{\prime}}^{r_{S}} 1\right)}\left\{\sum_{\theta_{i}=0}^{2 \pi} \sum_{r_{i}=0}^{r_{S}} E_{I}\left(\frac{N r_{i}^{\alpha}}{P_{t}}\right)\right. \\
& \exp \left(\frac{N r_{i}^{\alpha}}{P_{t}}\right)-\sum_{\theta_{i}=0}^{\theta_{\max }^{\prime}} \sum_{r_{i}=r_{\text {min }}^{\prime}}^{r_{S}} E_{I}\left(\frac{N d_{S R}^{\alpha}}{P_{t}}+\frac{N r_{i}^{\alpha}}{\mu P_{t}}\right) \\
& \left.\exp \left(\frac{N d_{S R}^{\alpha}}{P_{t}}+\frac{N r_{i}^{\alpha}}{\mu P_{t}}\right)\right\},
\end{aligned}
$$

$$
\begin{aligned}
= & \frac{1}{\ln 2\left(2 \pi r_{S}-\theta_{\text {max }}^{\prime}\left(r_{S}+1\right)+\sum_{\theta_{i}=0}^{\theta_{\text {max }}^{\prime}} \sqrt{r_{R}^{2}+d_{S R}^{2}-2 r_{R} d_{S R} \cos \theta_{i}}\right)} \\
& \left\{\sum_{\theta_{i}=0}^{2 \pi} \sum_{r_{i}=0}^{r_{S}} E_{I}\left(\frac{N r_{i}^{\alpha}}{P_{t}}\right) \exp \left(\frac{N r_{i}^{\alpha}}{P_{t}}\right)-\sum_{\theta_{i}=0}^{\theta_{\text {max }}^{\prime}} \sum_{r_{i}=r_{\text {min }}^{\prime}}^{r_{S}}\right. \\
& E_{I}\left(\frac{N d_{S R}^{\alpha}}{P_{t}}+\frac{N r_{i}^{\alpha}}{\mu P_{t}}\right) \\
& \left.\exp \left(\frac{N d_{S R}^{\alpha}}{P_{t}}+\frac{N r_{i}^{\alpha}}{\mu P_{t}}\right)\right\},
\end{aligned}
$$

here, $r_{\min }^{\prime}=\sqrt{r_{R}^{2}+d_{S R}^{2}-2 r_{R} d_{S R} \cos \theta_{i}}$, and $\theta_{\max }^{\prime}=$ $\cos ^{-1}\left[\frac{r_{R}^{2}+d_{S R}^{2}-r_{S}^{2}}{2 r_{R} d_{S R}}\right]$.

In (18), $\left(\sum_{\theta_{i}=0}^{\theta_{\max }^{\prime}} \sum_{r_{i}=r_{\text {min }}^{\prime}}^{r_{S}} 1\right)$ presents the total number of user locations in the area covered by the source only, and $\left(E_{I}\left(\frac{N d_{S R}^{\alpha}}{P_{t}}+\frac{N r_{i}^{\alpha}}{\mu P_{t}}\right) \exp \left(\frac{N d_{S R}^{\alpha}}{P_{t}}+\frac{N r_{i}^{\alpha}}{\mu P_{t}}\right)\right)$ presents the ergodic capacity of a given user location defined by $\left(\theta_{i}, r_{i}\right)$ and is derived in [3]. By averaging the corresponding ergodic capacity expressions of all the possible user locations in the area covered by the source only, $\bar{C}_{D L 1}$ can be presented as shown in (18). In (19), $\left(\sum_{\theta_{i}=0}^{2 \pi} \sum_{r_{i}=0}^{r_{S}} 1-\sum_{\theta_{i}=0}^{\theta_{\max }^{\prime}} \sum_{r_{i}=r_{\text {min }}^{\prime}}^{r_{S}} 1\right)$ presents the total number of user locations in the area covered by the source and the relay. Similar to (18), $\bar{C}_{D L 2}$ is presented in (19) as the average of the ergodic capacity expressions of all the user locations in the area covered by the source and the relay.

\section{BRUS scheme}

BRUS scheme was presented in [5] to enhance the spectral efficiency by using simultaneous transmissions for the source and the relay for user $U_{n}$ with probability $P_{S T}(n)$. $P_{S T}(n)$ is based on selecting a user $U_{n+1}$ located in the area covered by the source only to prevent the interference at this user from the relay. In order to average over all possible user selections, a binomial distribution should be used to define $P_{S T}(n)$ as follows:

$$
P_{S T}(n)=\sum_{k=1}^{N_{U}-n}\left(\begin{array}{l}
N_{U}-n \\
k
\end{array}\right) P_{D L 1}^{k}\left(1-P_{D L 1}\right)^{N_{U}-n-k},
$$

where, $N_{U}$ is the number of users per frame. As detailed in [5], $P_{S T}\left(N_{U}\right)=P_{S T}\left(N_{U}-1\right)$ because the relaying transmission for user $N_{U}$ can affect user $U 1$ only at the first time slot of the following frame, i.e., the probability that there are simultaneous transmissions of the source and the relay for user $N_{U}$ is the same as for user $N_{U}-1$. Based on the derivation of the ergodic capacity in [5], the average 
ergodic capacity expression for BRUS scheme is given by the following:

$$
\begin{aligned}
\bar{C}^{\text {BRUS }}= & P_{D F}\left[\frac{1}{N_{U}} \sum_{n=1}^{N_{U}} P_{S T}(n) 2 \bar{C}_{D F}\right. \\
& \left.+\left(1-\frac{1}{N_{U}} \sum_{n=1}^{N_{U}} P_{S T}(n)\right) \bar{C}_{D F}\right] \\
& +P_{D L} \bar{C}_{D L} .
\end{aligned}
$$

\section{EASE metric}

Based on the expressions of the average ergodic capacity for each scheme $S \in\{H D R, I C R S, B R U S\}$, the average affected area, and the SRCndx, the new $E A S E^{S}$ metric is introduced as follows:

$$
E A S E^{S}=\operatorname{SRCndx} \frac{\bar{C}^{S}}{\bar{A}} .
$$

where, the new metric $\operatorname{EASE}^{S}$ depends on three elements, the new proposed metric $S R C n d x$, the average ergodic capacity $\bar{C}^{S}$, and the total affected area $\bar{A}$. $\bar{C}^{S}$ and $\bar{A}$ depend on the transmission power $\left(P_{t}\right)$, where they increase as $P$ increases. Consequently, for a given value of $P_{t}>1, \bar{C}^{S}$ will remain constant, $\bar{A}$ will increase, and hence the EASE will decrease. In another scenario, by using a small value of $P_{t}, \bar{C}^{S}$ and $\bar{A}$ will be decreased. The minimum and maximum value of $P_{t}$ is limited by $S R C n d x$, where it will give zero if there is no need for cooperative transmission, and one when relaying transmission is beneficial. Hence, $S R C n d x$ defines the range of transmission power, where cooperative transmission is needed, and $\frac{\bar{C}^{S}}{\bar{A}}$ presents the tradeoff between $\bar{C}^{S}, \bar{A}$, and $P_{t}$. Finally, the best transmission power that maximizes the EASE metric can be numerically selected and used to offer the best transmission quality in terms of coverage area and spectrum efficiency.

\section{Numerical results and interpretations}

We present in this section the EASE performance analysis of DL and DF communication systems as well as a comparison of the proposed EASE and GASE [3] metrics. In addition, a comparison of EASE performances for the different interference management schemes is presented. The parameters of the simulations are the following: $d_{S R}=500 \mathrm{~m}, \mu=0.5, P_{\min }=-90 \mathrm{dBm}, \alpha=3,4,5$, and $6, N=-100 \mathrm{dBm}$, and $N_{U}=20$.

In Fig. 8, we present the EASE metric variations versus $P_{t}$ for DL and DF relay systems. To make a fair comparison, we have used the same average affected area for DL and DF, which is the area covered by the source and the relay; hence, the EASE result of DL is presented in the range of power where SRCndx is equal to one to make sure that the nodes are communicating. Simulation results show that the EASE metric is equal to zero for some values of $P_{t s}$, when there is no beneficial communication between the source and the relay, i.e., there is no communication between the source and the relay or there is no need of relaying. For the effective range of $P_{t}$, where EASE metric is not zero, the new metric describes the area spectral efficiency of the communication system by giving the number of transmitted bits per time unit via frequency unit in coverage area unit. The outperformance of DF in term of EASE when compared to DL transmissions is clear. The best transmission power that maximizes the EASE can be numerically selected and used to offer the best transmission quality in term

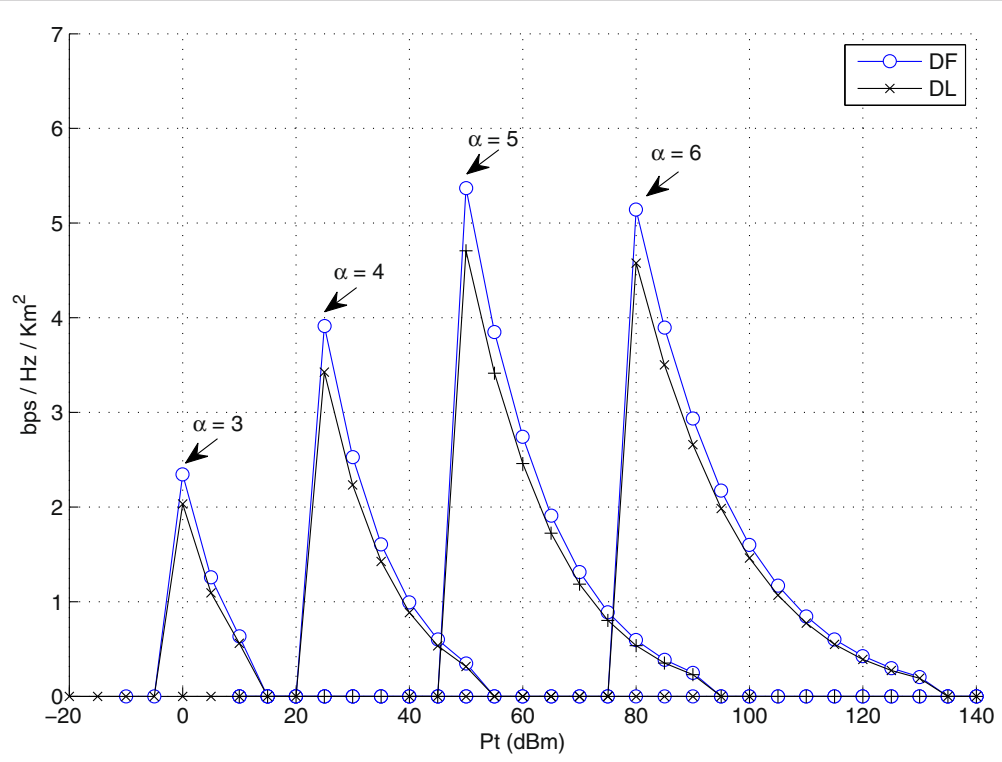

Fig. 8 Comparisons of EASE performances for DL and DF relaying systems 


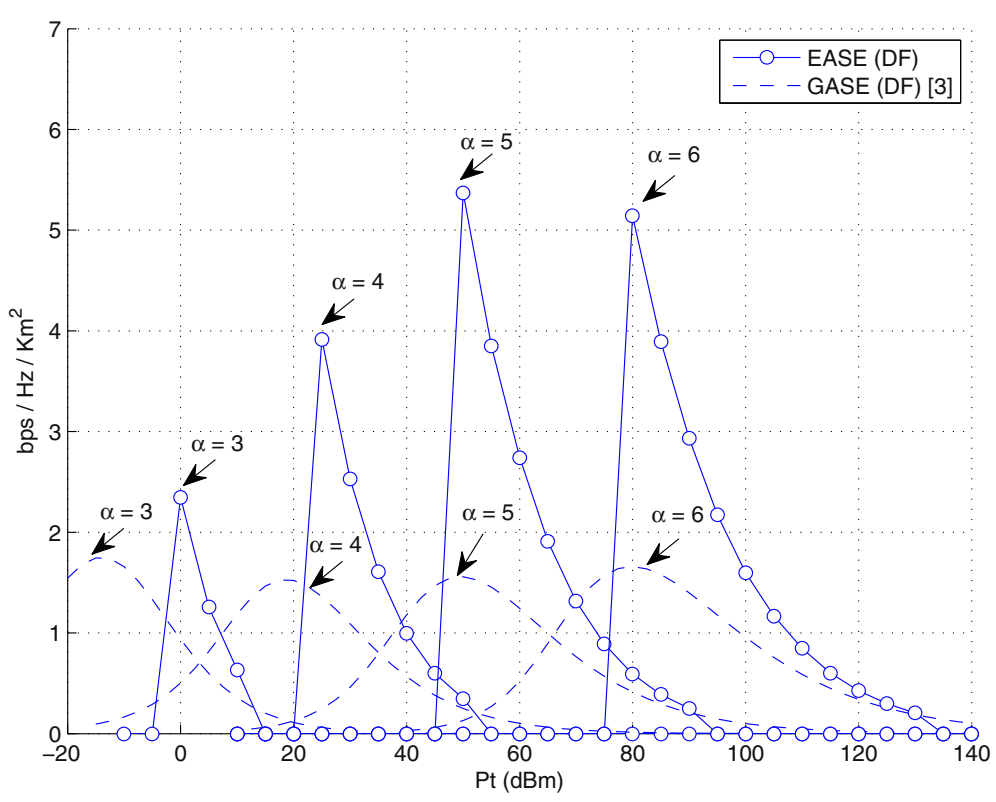

Fig. 9 EASE vs. GASE for DF relay systems

of coverage area and spectrum efficiency for a given scenario.

In Fig. 9, a comparison of the proposed EASE and GASE [3] metrics for DF communications is presented. As mentioned in the "Introduction" section and as shown in this figure, the maximization of GASE was presented in [3] without power transmission constraint which may result in an optimal value of transmission power that maximizes the GASE but with received signal power less than $P_{\text {min }}$. For example, as shown in this figure and for $\alpha=3$, the optimal value of $P_{t}$ which maximizes GASE metric is equal to $-15 \mathrm{~dB}$. According to (3), this value does not guarantee a received power at the destination which is greater than $P_{\text {min }}$. Note that the proposed index SRCndx for this power is equal to zero.

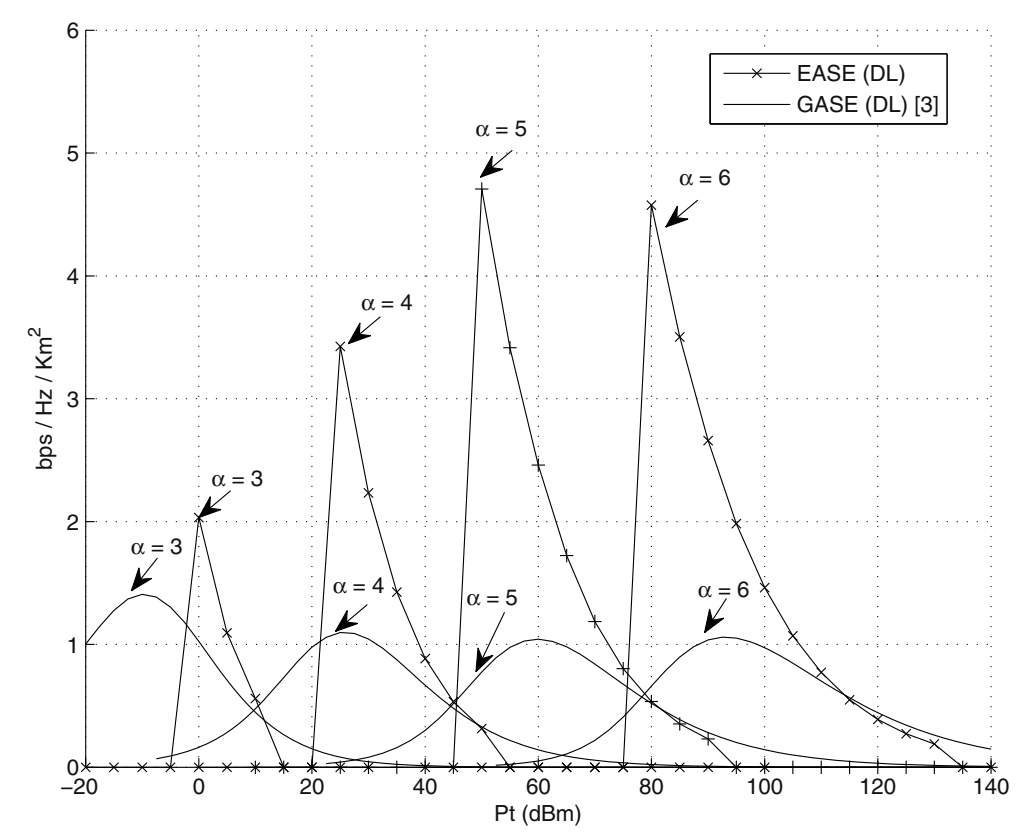

Fig. 10 EASE vs. GASE for DL transmissions 


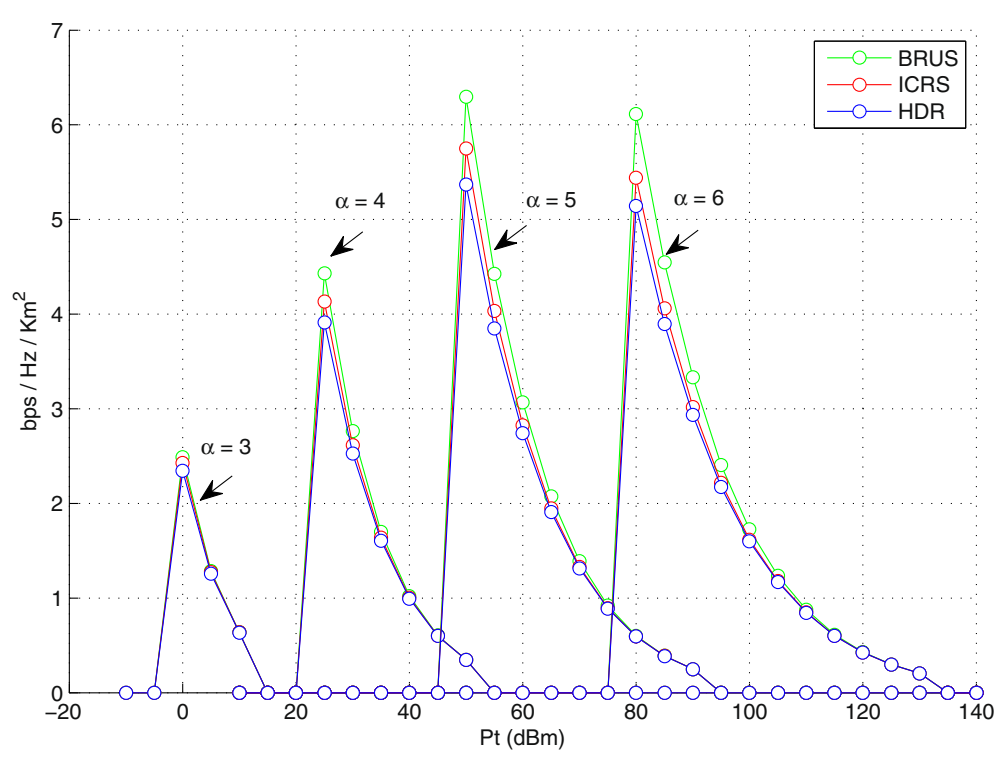

Fig. 11 EASE vs. $P_{t}$ for different interference management schemes

The power transmission constraint problems in [3] is confirmed by Fig. 10, where a comparison of the proposed EASE and GASE [3] metrics for DL communications is presented. This power constraint problem is resolved by the proposed new parameter SRCndx that eliminates the $P_{t}$ values from the maximization range, where the communications between the source and the destination are useless. This is confirmed in Figs. 9 and 10 for the proposed EASE metric performances.

Figure 11 shows the comparison of the EASE metric for the different interference management schemes. It is clear here that BRUS and ICRS schemes outperform the HDR scheme with better performance of BRUS scheme. This is because, within HDR scheme, each cooperative transmission needs two time slots, i.e., there is no simultaneous transmissions from the relay and the source to prevent the interference at the receiver. Hence, the spectral efficiency will be divided by two, and consequently, the EASE will decrease in value. However, the simultaneous transmission which prevent the loss of frequency efficiency is possible for BRUS and ICRS schemes for some cases. As shown in Fig. 11, the EASE performance for BRUS scheme is better than ICRS scheme. This is because the probability of the simultaneous transmission within BRUS scheme is higher. In fact, the probability to find a user satisfying the interference constraint within BRUS scheme is higher than the probability when user $n$ and $n+1$ are randomly located, and user $n+1$ does not receive interference from the relay.

\section{Conclusions}

In this paper, a new performance metric, EASE, has been introduced to quantify the spectral efficiency as well as the spatial property for point-to-point and DF relayed cellular transmission systems with interference management. For given transmissions parameters, the EASE metric can describe the area spectral efficiency by giving the number of transmitted bits per time-frequency unit in coverage area unit. The new metric is based on the average affected area, the average ergodic capacity, and a new proposed parameter SRCndx. SRCndx can validate the communication possibility between a source and a relay for given transmission parameters as well as the necessity of using cooperative communications. Numerical examples have shown that the EASE metric provides a new perspective on the design of wireless communication systems, especially on the transmitting power selection.

\section{Competing interests}

The authors declare that they have no competing interests.

\section{Acknowledgements}

This paper was made possible by NPRP grant 4-1119-2-427 from the Qatar National Research Fund (a member of Qatar Foundation). The statements made herein are solely the responsibility of the authors.

\section{Author details}

${ }^{1}$ Electrical Engineering Department, Qatar University, Doha, Qatar. ${ }^{2}$ Wireless Intelligent Networks Center, Nile University, Giza, Egypt. ${ }^{3}$ Electronics and

Communications Department, Cairo University, Doha, Qatar.

Received: 15 November 2014 Accepted: 3 August 2015

Published online: 18 August 2015

\section{References}

1. Q Li, RQ Huand, Y Qian, G Wu, Cooperative communications for wireless networks, techniques and applications in LTE advanced systems. IEEE Wireless Commun. Mag. 19(2), 22-29 (2012)

2. M Rumney, LTE and the Evolution to $4 G$ Wireless Design and Measurement Challenges. (Agilent Technologies Publication, USA, 2012), pp. 1-10 
3. L Zhang, HC Yang, MO Hasna, Generalized area spectral efficiency: an effective performance metric for green wireless communications. IEEE Trans. Commun. 99, 1-11 (2014)

4. TChen, H Kim, Y Yang, Energy Efficiency Metrics for Green Wireless Communications. (Wireless Communications and Signal Processing (WCSP), 2010 International Conference, Suzhou, 2010), pp. 1-6

5. A Omri, MO Hasna, Novel Cooperative Communication Schemes with Interference Management for Multi-User Wireless Networks.

(Communications (ICC), 2013 IEEE International Conference, Budapest, 2013), pp. 5651-5656

6. A Omri, MO Hasna, Performance Analysis of OFDMA Based Wireless Cooperative Networks with Interference Management. (Vehicular Technology Conference (VTC Spring), 2013 77th IEEE, Dresden, 2013), pp. 1-6

7. H Chamkhia, MO Hasna, Sub-Channels Selection Schemes with Interference Management for Underlay Cognitive Networks. (Personal Indoor and Mobile Radio Communications (PIMRC), 2013 IEEE 24th International Symposium, London, United Kingdom, 2013), pp. 3016-3021

8. TC Clancy, Achievable Capacity Under the Interference Temperature Model. (INFOCOM 2007. 26th IEEE International Conference on Computer Communications. IEEE, Anchorage, AK, 2007), pp. 794-802

9. ETSI, Energy Efficiency of Wireless Access Network Equipment. TS102706 (2009). Available: http://www.etsi.org

10. F Richter, AJ Fehske, GP Fettweis, Energy Efficiency Aspects of Base Station Deployment Strategies for Cellular Networks. (Vehicular Technology Conference Fall (VTC 2009-Fall), 2009 70th IEEE, Anchorage, AK, 2009), pp. $1-5$

11. Y Chen, S Zhang, S Xu, GY Li, Fundamental Trade-Offs on Green Wireless Networks. IEEE Com. Mag. 49(6), 30-37 (2011)

12. AJ Fehske, P Marsch, GP Fettweis, Bit Per Joule Efficiency of Cooperating Base Stations in Cellular Networks. (GLOBECOM Workshops (GC Wkshps), 2010 IEEE, Miami, FL, 2010), pp. 1406-1411

13. J Lorincz, N Dimitrov, T Matijevic, Bit Per Joule and Area Energy Efficiency of Heterogeneous Macro Base Station Sites. (Software, Telecommunications and Computer Networks (SoftCOM), 2012 20th International Conference, Split, 2012), pp. 1-6

14. MS Alouini, AJ Goldsmith, Area Spectral Efficiency of Cellular Mobile Radio Systems. IEEE TVT. 48(4), 1047-1066 (1999)

15. L Zhang, HC Yang, MO Hasna, Generalized Area Spectral Efficiency: An Effective Performance Metric for Green Wireless Communications. (Communications (ICC), 2013 IEEE International Conference, Budapest, 2013), pp. 5376-5380

16. L Zhang, MO Hasna, HC Yang, Area Spectral Efficiency of Cooperative Network with Opportunistic Relaying. (Vehicular Technology Conference (VTC Spring), 2012 75th IEEE, Yokohama, 2012), pp. 1-5

17. A Omri, MO Hasna, Interference Management Schemes with General Order Statistics and Interference Constraint for Multi-User Cooperative Wireless Networks. (Global Communications Conference (GLOBECOM), 2013 IEEE, Atlanta, GA, 2013), pp. 1779-1784

18. IS Gradshteyn, IM Ryzhik, Table of Integrals, Series, and Products, 7th edn. (Elsevier Academic Press, USA, 2007)

\section{Submit your manuscript to a SpringerOpen ${ }^{\circ}$ journal and benefit from:}

- Convenient online submission

- Rigorous peer review

- Immediate publication on acceptance

- Open access: articles freely available online

- High visibility within the field

- Retaining the copyright to your article

Submit your next manuscript at $\boldsymbol{\wedge}$ springeropen.com 